\title{
Complementary Cumulative Distribution Function for Performance Analysis of OFDM Signals
}

\author{
Parneet Kaur ${ }^{1}$, Ravinder Singh ${ }^{2}$ \\ ${ }^{1}$ (University College of Engineering/ Punjabi University, India) \\ ${ }^{2}$ (Dr. B.R. Ambedkar National Institute of Technology / India)
}

\begin{abstract}
Peak-to-Average Power Ratio (PAPR) is an important parameter for the analysis of Orthogonal frequency division multiplexing (OFDM) signals. CCDF curves are known to have great importance in the study of signal performance on the basis of power level. This paper presents the CCDF performance of the OFDM signal with different number of carriers. Simulation is used to implement the CDF equation and its accuracy is checked on the results. Theoretical results and simulation results are compared.
\end{abstract}

Key words-Crest factor, CCDF, OFDM, PAPR, and PDF.

\section{Introduction}

OFDM systems are known to have a high PAPR (Peak-to-Average Power Ratio) because the transmit signals in an OFDM system can have high peak values in the time domain since many subcarrier components are added via an IFFT operation. Decreasing the SQNR (Signal-to-Quantization Noise Ratio) of ADC (Analogto-Digital converter) and DAC (Digital-to-Analog Converter) while degrading the efficiency of the power amplifier in the transmitter the high PAPR is considered one of the most detrimental aspects in the OFDM system. In the uplink the efficiency of power amplifier is critical due to the limited battery power in a mobile terminal hence the PAPR problem is more important.[1] A non linear distortion is imposed in linear amplifiers on their outputs due to their saturation characteristics caused by an input much larger than its nominal value.

The input-output characteristics of high power amplifier (HPA) in terms of the input power $\boldsymbol{P}_{\text {in }}$ and the output power $P_{\text {out }}$ in figure 1. The maximum possible output is limited by $P_{i n}^{\max }$ when the corresponding input power is given by $P_{\text {out }}^{\max }$ due to the aforementioned saturation characteristic of the amplifier. The input power must be backed off so as to operate in the linear region as illustrated in Figure 1, describing the nonlinear region by Input Back-Off (IBO) or Output Back-Off (OBO) [2]:

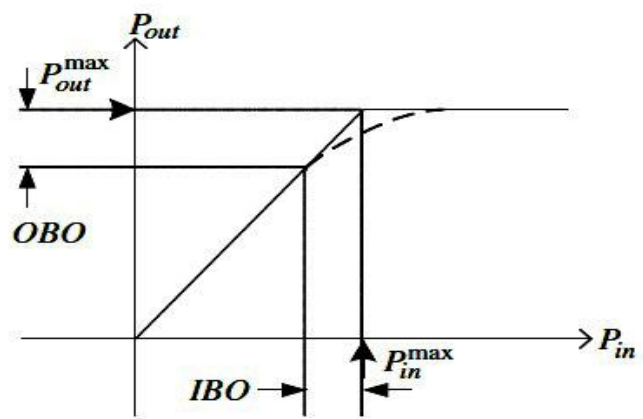

Fig.1 Input- Output characteristic of an HPA

$$
I B O=10 \log _{10} \frac{P_{\text {in }}^{\max }}{P_{\text {in }}}, O B O=10 \log _{10} \frac{P_{\text {out }}^{\max }}{P_{\text {out }}}
$$

\section{CCDF PLOTS}

A CCDF curve shows how much time the signal spends at or above a given power level. The power level is expressed in $\mathrm{dB}$ relative to the average power. A CCDF curve is basically a plot of relative power levels versus probability. [5,6] Mathematically CCDF can be explained with a set of data having the probability density function (PDF). To obtain the Cumulative Distribution Function (CDF), the integral of the PDF is computed. Then inverting the CDF results in the CCDF. It concludes that the CCDF is the complement of the $\mathrm{CDF}$ or $\mathrm{CCDF}=1-\mathrm{CDF}$. 


\section{MATHEMATICAL ANALYSIS OF PAPR USING CCDF}

PAPR is the ratio between the maximum power and the average power of the complex pass band signal $\tilde{s}(t)$ [7], that is,

$$
\begin{aligned}
& \operatorname{PAPR}\left\{\tilde{s}_{I}(t)\right\}=\frac{\max \mid \operatorname{Re}\left(\left.\tilde{s}_{I}(t) e^{j 2 \pi f_{c} t}\right|^{2}\right.}{E\left\{\mid \operatorname{Re}\left(\left.\tilde{s}_{I}(t) e^{j 2 \pi f_{c} t}\right|^{2}\right\}\right.} \\
& \operatorname{PAPR}\left\{\tilde{s}_{I}(t)\right\}=\frac{\max \left|\tilde{s}_{I}(t)\right|^{2}}{E\left\{\left|\tilde{s}_{I}(t)\right|^{2}\right\}}
\end{aligned}
$$

The above power characteristics can also be described in terms of their magnitudes (not power) by defining the crest factor (CF) [4] as

Pass band condition: $C F=\sqrt{P A P R}$

Baseband condition: $C F=\sqrt{P M E P R}$

In order to find the probability that the signal power is out of the linear range of the HPA, the distribution of output signals for IFFT in the OFDM system is considered first. It is assumed that the real and imaginary parts of the time-domain complex OFDM signal $s(t)$, at the transmitter after IFFT, have asymptotically Gaussian distributions for a sufficiently large number of subcarriers by the central limit theorem, at the same time as the input signals of N-point IFFT have the independent and finite magnitudes which are uniformly distributed for QPSK and QAM. After that the amplitude of the OFDM signal $s(t)$ follows a Rayleigh distribution. Let $\left\{Z_{n}\right\}$ be the magnitudes of complex samples $\left\{\left|s\left(n T_{s} / N\right)\right|\right\}_{n=0}^{N-1}$. It is assumed that the average power of $s(t)$ is equal to one, that is, $E\left\{\left|\tilde{s}_{I}(t)\right|^{2}\right\}=1$, then $\left\{Z_{n}\right\}$ are the i.i.d. Rayleigh random variables normalized with its own average power, which has the following probability density function:

$$
f z_{n}(z)=\frac{z}{\sigma^{2}} e^{\frac{z^{2}}{2 \sigma^{2}}}=2 z e^{-z^{2}}, n=0,1,2, \ldots \ldots . N-1
$$

The maximum of $Z_{n}$ is equivalent to the crest factor (CF) defined in Equation (3). Let $Z_{\max }$ denote the crest factor (i.e., $Z_{\max }=\max _{n=0,1, \ldots N-1} Z_{n}$ Now, the cumulative distribution function (CDF) of $Z_{\max }$ is given as

$$
\begin{aligned}
& F z_{\max }(z)=P\left(Z_{\max }<z\right) \\
& =P\left(Z_{o}<z\right) \cdot P\left(Z_{1}<z\right) \ldots . . P\left(Z_{N-1}<z\right) \\
& =\left(1-e^{-z^{2}}\right)^{N}
\end{aligned}
$$

where $P\left(Z_{n}<z\right)=\int_{0}^{z} f z_{n}(x) d x, n=0,1,2, \ldots N-1$.

In order to find the probability that the crest factor (CF) exceeds $\mathrm{z}$, we consider the following complementary $\mathrm{CDF}(\mathrm{CCDF})$ :

$$
\begin{aligned}
\tilde{F} z_{\max }(z) & =P\left(Z_{\text {max }}>z\right) \\
& =1-P\left(Z_{\text {max }} \leq z\right) \\
& =1-F z_{\text {max }}(z) \\
& =1-\left(1-e^{-z^{2}}\right)^{\alpha N}
\end{aligned}
$$

The following simplified CDF will be used as it is difficult to derive the exact CDF for the oversampled signals, $F_{Z}(z) \approx\left(1-e^{-z^{2}}\right)^{\alpha N}$

where $\alpha$ has to be determined by fitting the theoretical CDF into the actual one [3]. 


\section{Simulation Results}

Simulation has been done for different number of carriers using MATLAB. In fig. 2 the theoretical and simulated CCDFs of OFDM signals are shown with $\mathrm{N}=64,128,256,512,1024$. It also provides with $\alpha=2.8$ as an appropriate value for sufficiently large value of $\mathrm{N}$. The PAPR values for different number of carriers are obtained for theoretical as well as simulation perspectives.

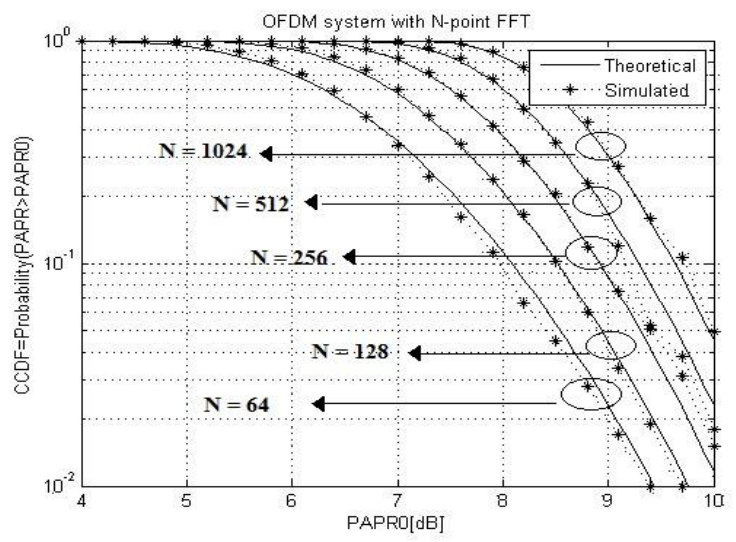

Fig 2 CCDFs of OFDM signal with $\mathrm{N}=64,128,256,512$ and 1024

\section{Conclusions}

The simulation results help to formulate the conclusion that the simulated values of CCDFs deviate from the theoretical ones as $\mathrm{N}$ becomes small. With the increasing values of $\mathrm{N}$ the deviation is increasing. CCDF plots provide an efficient method for the analysis of the PAPR of the OFDM signal. The equation for the $\mathrm{CDF}$ of the signals is accurate only when $\mathrm{N}$ is sufficiently large.

\section{REFERENCES}

[1] Evegenii Krouk, Sergei Semnov , "Modulation and coding techniques in wireless communications", 2011.

[2] Yong soo cho, Jaekwon Kim, Won young Yang and Chung G. Kang, MIMO- OFDM wireless communication with MATLAB. John Wiley \& Sons. (2010).

[3] Van Nee, R. and deWild, A “Reducing the peak-to-average power ratio of OFDM.” IEEE VTC'98, vol.3, pp. 18-21, .May 1998.

[4] Ashraf A. Eltholth, Adel R. Mekhail, A.Elshirbini, M.I. Dessouki , A.I. Abdelfattah, " Low crest factor modulation techniques for orthogonal frequency division multiplexing (OFDM)",UBICC, Ubiquitous computing and communication Journel, Vol 4, 2007.

[5] Ming kang, Lin Yang, Mohamed-Slim Alouini, "Capacity of MIMO Ricean channels with multiple correlated Rayleigh co-channel interferers.", IEEE , pp 2636-2647, Oct. 2003.

[6] Yonglan Zhu, pooi yuen kam, Yan Xin, “ On the mutual information distribution of MIMO Ricean fading channels.”, IEEE Trans. Communications, May 2009.

[7] Palicot, J. and Louet,Y. Power ratio definitions and analysis in single carrier modulations. EUSIPCO, Antalya, Turkey, Sep. 2005. 